\title{
Article
}

\section{Développement durable et disciplines scientifiques}

\author{
Edwin Zaccaï \\ Ingénieur physicien, philosophe, Institut de gestion de l'environnement et d'aménagement du territoire, Université Libre de \\ Bruxelles, CP 130/02, Avenue F. Roosevelt, 50, 1050 Bruxelles, Belgique
}

La notion de développement durable a-t-elle un sens pour la recherche? Pour la formation ? S'agit-il d'une nouvelle opportunité pour faire accepter des propositions dans les appels à projet? De la génération de nouvelles disciplines ou de nouveaux arrangements interdisciplinaires? De l'émergence de nouvelles pratiques de recherche, plus intégrées, plus ouvertes sur les partenariats avec des non-chercheurs? Six ans après la parution de l'ouvrage Le Développement durable, de l'utopie au concept : de nouveaux chantiers pour la recherche, dans la collection Elsevier-NSS, Edwin Zaccaï nous fait part de ses propres réflexions sur la question.

La Rédaction

\section{Mots-clés :}

développement durable ; recherche ; épistémologie ; disciplines académiques ; enseignement

\section{Keywords:}

sustainable development; research; epistemology; academic disciplines; teaching

\begin{abstract}
Résumé - Cet article explore le positionnement du développement durable par rapport aux disciplines scientifiques. Il part d'une confrontation des caractéristiques des recherches académiques en matière de développement durable avec les critères définis par T. Kuhn pour les disciplines, les paradigmes et les révolutions scientifiques. Selon ces références, le développement durable n'apparaît pas comme un paradigme général, mais, réinterprété diversement par les disciplines, il introduit des modifications dans les objets et les méthodes de recherche. Dans un deuxième temps, des conceptions plus récentes des disciplines sont utilisées, selon lesquelles les interactions avec des processus situés hors des communautés scientifiques se révèlent essentielles. On suggère alors que l'une des modalités de recherche en matière de développement durable, davantage en termes de démarche que de contenu, pourrait apparaître comme une tentative d'assumer consciemment les inscriptions sociales et politiques de pratiques scientifiques et d'en tirer des conséquences. Certaines implications de ces différentes analyses pour l'enseignement universitaire sont présentées en conclusion.
\end{abstract}

\begin{abstract}
Sustainable development and scientific disciplines. This paper analyses how the concept of sustainable development interacts with scientific disciplines. First it confronts the characteristics of academic research dealing with sustainable development to the criteria formulated by T. Kuhn for disciplines, paradigms and scientific revolutions. In this framework, sustainable development does not appear as an overarching paradigm. It is interpreted differently within several disciplines, and can modify their subjects and methods of research. In the second part of the paper, more recent theory about scientific disciplines is introduced, underlining the importance of interactions with spheres external to scientific communities. The paper suggests that a major feature of sustainable development research - that has more to deal with methods than with the subjects studied - would be to deliberately assume the interactions of science, society and policy, and to deal with their consequences. We conclude with some implications of these analyses for teaching sustainable development in higher education.
\end{abstract}

Auteur correspondant : ezaccai@ulb.ac.be

E. Zaccaï, également docteur en environnement, est chargé de cours à l'Université Libre de Bruxelles (ULB) et chargé d'enseignement à Sciences Po (Paris); il est aussi directeur du Centre d'études du développement durable à l’ULB. 


\section{Développement durable et milieux académiques}

Comme chacun peut le vérifier, la diffusion des références au développement durable s'est poursuivie ces dernières années dans les sphères de la politique, des médias ou encore des entreprises, mais aussi de la recherche et de l'enseignement ${ }^{1}$. Avec la multiplication de ces références, les critiques qui accompagnent ce concept depuis son origine dans les années 1980 s'accroissent. On connaît les possibilités d'opportunisme des renvois à une notion large et consensuelle, porteuse d'une promesse de renouvellement positif, sans pour autant conduire à des changements à la mesure des enjeux évoqués (Smouts, 2005; Aubertin et Vivien, 2006). Il n'en reste pas moins que ces références contribuent à induire des modifications dans les structurations de certains champs politiques et professionnels. L'objet de cet article est d'examiner certains de ces changements dans le domaine académique de la recherche et de l'enseignement supérieur, à travers un prisme particulier, celui des disciplines scientifiques. Les disciplines scientifiques structurent fortement l'enseignement supérieur, ainsi que la formation et la production des chercheurs. C'est pourquoi nous nous proposons de relever avec quelque précision le positionnement du développement durable par rapport à ces structures, car ces interactions vont conditionner son inscription et son influence éventuelle dans le monde académique.

Dans cet article, la référence première utilisée pour décrire les caractéristiques des disciplines scientifiques est celle du théoricien qui a marqué leur définition contemporaine, Thomas Kuhn. Nous reprendrons, dans une première partie, des éléments-clés des définitions utilisées par celui-ci, pour les confronter à celles du développement durable. Toutefois, ces conceptions de Kuhn ont été plus particulièrement développées en regard des sciences exactes durant les années 1960 ; d'autre part, la sociologie des sciences a conduit ultérieurement à relativiser la stabilité interne des disciplines ainsi que leur relative autonomie en regard d'acteurs non scientifiques. Ces enrichissements seront considérés dans un deuxième temps en relation avec le développement durable. Plutôt que $\mathrm{d}$ 'avoir recours à une définition unique et stable des disciplines scientifiques, nous utiliserons ces différents éclairages, qui chacun vont offrir des éléments à la question centrale de cet article, à savoir le positionnement du développement durable par rapport à ces structures, même si elles sont diverses (disciplines, sous-disciplines) et mouvantes (évolutions historiques).

\footnotetext{
${ }^{1}$ Je remercie le Commissariat général du pavillon de France de l'exposition d'Aichi pour son invitation à traiter de ce thème lors d'un colloque en septembre 2005. Cet exposé est à l'origine du présent article.
}

\section{Définitions du développement durable}

Le développement durable dans le monde académique et de la recherche renvoie à des transformations (associées à « développement ») incluant en premier lieu, mais pas uniquement, des critères de protection de l'environnement («durable»). Il n'en reste pas moins que des questions se posent à chaque pas sur la portée des différents termes, tant il est vrai que, comme le remarquait dès 1988 O'Riordan, le développement durable paraît avoir été inventé pour que des «successions sans fin de scientifiques et de diplomates puissent passer de longues et confortables heures à essayer de le définir avec succès » (cité par Reid, 1995, p. 221).

Outre les discussions sur la portée des différents termes mentionnés plus haut, il faut également voir que même cette délimitation minimale n'est pas pour autant acquise. Ainsi, pour les grandes entreprises qui se réfèrent au développement durable, l'un des champs les plus actifs aujourd'hui autour de cette notion, l'environnement, $n$ 'est pas la notion centrale : c'est de "responsabilité sociale» (ou «sociétale ) qu'il s'agit, avec un triptyque formé de $l^{\prime}$ «environnemental», du «social» et de «l'économique $»^{2}$. Il en va de même, d'ailleurs, dans certains domaines du champ politico-administratif européen, où la procédure importante des études d'impacts (sur le développement durable) des directives et des politiques place sur le même pied des évaluations économiques, sociales et environnementales (Commission des Communautés européennes, 2002). Quant aux Agendas 21 locaux, leur notion centrale est davantage celle de gouvernance participative que de protection de l'environnement. Dans le domaine de la recherche, un important rapport - sur lequel nous reviendrons - a défini en France les relations avec le développement durable en indiquant : «Il est utile de souligner les traits distinctifs résultant de la conjonction de l'idée de développement et de l'idée de durabilité : le temps long, l'espace élargi et les interconnexions » (Hautcœur, 2003, p. 5). Néanmoins, dans le rapport même, les questions environnementales prédominent ${ }^{3}$.

En examinant un éventail de références au développement durable, nous avons proposé (Zaccaï, 2002) de retenir les cinq caractéristiques suivantes comme étant le plus souvent réunies : importance donnée à l'environnement ;

\footnotetext{
${ }^{2}$ Voir à ce sujet les grilles d'indicateurs du Global Reporting Initiative, une organisation internationale qui propose des procédures pour la publication de rapports sur ces sujets. En France, la loi sur les nouvelles régulations économiques (NRE) de février 2002 oblige les grandes entreprises à publier des informations sur les conséquences sociales, territoriales et environnementales de leurs activités. En 2005-2006, plus de $90 \%$ des grandes entreprises auraient publié un rapport spécifique sur le développement durable, produit par des équipes dédiées à cet effet (Mamou, 2006).

${ }^{3}$ Le rapport a d'ailleurs été publié en 2005 dans la collection «Réponses environnement » à la Documentation française.
} 
vision à long terme ; vision mondiale; intégration des dimensions du développement; affirmation de la nouveauté du projet ${ }^{4}$. La dernière citée devrait être comprise comme une métacaractéristique, qui fait partie aussi de son intérêt, assez souvent d'ailleurs pour suggérer que le développement durable est en phase avec les évolutions les plus récentes et nécessaires. Nous en verrons des exemples tant dans le domaine de la recherche que dans celui de l'enseignement.

Il peut sembler incommode de s'avancer sur le terrain de l'exploration du positionnement du développement durable par rapport aux disciplines scientifiques avec un ensemble de départ aussi flou. Néanmoins, il correspond aux façons diverses dont des références au développement durable vont être pour ainsi dire injectées à différents lieux de l'enseignement et de la recherche : dans une école de commerce ou dans un institut de recherche en chimie, les références seront en effet bien diversifiées. Cela ne nous empêchera pas de préciser, en cours d'analyse, des caractéristiques plus précises et délimitées quand il y a lieu - en particulier sur les références plus affirmées au domaine de l'environnement et au positionnement de disciplines diverses à cet égard. Remarquons aussi que ces disciplines scientifiques ont contribué avec un éventail d'acteurs sociaux et politiques à la multiplicité de ses définitions. Dans certaines définitions d'acteurs, comme celles des ONG environnementales de l'Union mondiale pour la nature (UICN), on décèle une nette influence biologique conservationniste, avec la notion de limites de capacités de charge ${ }^{5}$, tandis qu'il n'est pas difficile de retrouver la touche économiste dans les définitions soutenues par des acteurs industriels ou financiers.

\section{Le développement durable comme paradigme?}

\section{Concepts de base : Kuhn}

L'un des éléments-clés de la réflexion de T. Kuhn sur la science est la notion de communautés de scientifiques. Si des philosophes des sciences antérieurs à Kuhn, comme K. Popper ou des auteurs du positivisme logique, se concentraient en effet sur les critères de validité de la science en général, Kuhn s'intéresse plus particulièrement

\footnotetext{
${ }^{4}$ Il existe bien sûr de nombreuses autres séries de caractéristiques : voir des typologies dans Zaccaï (2002) et Hopwood et al. (2005).

5 Sur les filiations entre les approches de conservation, certaines remontant à plusieurs siècles, et la conceptualisation du développement durable, consulter Adams (2001, p. 22-79). Il y a toujours des distanciations possibles entre les approches écologistes naturalistes et les orientations résolument anthropocentriques du développement durable. Voir, par exemple, Newton et Freyfogle (2005).
}

à ce qui se passe dans des communautés disciplinaires de scientifiques, de personnes, donc, et différenciées.

Kuhn observe que les principales disciplines scientifiques ont leurs communautés bien établies : physiciens, chimistes, par exemple, mais aussi des sous-communautés comme les biochimistes ou les astrophysiciens. Les membres de ces communautés ont « tous eu une formation et une initiation professionnelle semblables, à un degré inégalé dans la plupart des autres disciplines. [...] ils ont assimilé la même littérature technique et en ont retiré dans l'ensemble le même enseignement » (Kuhn, 1983, p. 241).

«Certains critères, tels que la nature du plus haut diplôme obtenu, l'appartenance à des sociétés de spécialistes ou le fait de lire certains périodiques, sont généralement plus que suffisants » pour préciser cette appartenance à une communauté scientifique (ibid., p. 242). Derrière ces signes, une matrice disciplinaire est commune aux membres d'une discipline. Les paradigmes, une notionclé introduite par Kuhn, sont les éléments constituant cette matrice. Ils comprennent des éléments partagés, relativement non problématiques ou unanimes pour des professionnels d'une discipline. Les paradigmes peuvent aussi être vus comme "les solutions concrètes de problèmes que les étudiants rencontrent, dès le début de leur formation scientifique », par exemple comme sujets d'examen ou à la fin des chapitres des manuels (ibid., p. 254), et au « premier chef un paradigme régit, non un domaine scientifique, mais un groupe de savants » (ibid., p. 245$)^{6}$.

Kuhn insiste aussi sur une autre notion qui peut nous intéresser pour le développement durable, celle de révolutions scientifiques. Pour lui, ce sont d'abord des microrévolutions, qui peuvent se produire dans des groupes de quelques dizaines de personnes seulement, mais peuvent devenir aussi les ferments de changements de plus grande importance. L'un des aspects principaux de toute révolution est que certains des rapports entre les objets étudiés changent. «Des faits qui étaient groupés dans le même ensemble auparavant sont groupés ensuite dans des ensembles [scientifiques] différents » (ibid., p. 272). Dans une nouvelle configuration, induite par une révolution scientifique, un ancien paradigme est remplacé par un autre, qui n'est pas nécessairement commensurable ni compatible avec le précédent.

Nous nuancerons plus loin ces notions de base pour montrer que, selon des analyses plus récentes, les disciplines, même en situation de stabilité, sont moins

\footnotetext{
${ }^{6}$ Ces définitions plus restrictives que celles de son ouvrage de 1962, The Structure of Scientific Revolution, ont été formulées par Kuhn notamment en réponse à des critiques sur les notions initiales (Audi, 1995). Celles-ci n'ont cependant jamais été aussi larges que des usages communs actuels du mot paradigme, synonymes de visions du monde, de cadres de pensées, de valeurs, etc.
} 
cohérentes qu'il n'y paraît et surtout plus irriguées de processus issus des sphères hors science. Mais, dans cette première étape, tentons de situer le développement durable par rapport aux concepts kuhniens qui viennent d'être introduits.

\section{Réinterprétations du développement durable par les disciplines}

Première réflexion, le développement durable est réinterprété par différentes disciplines. Il est loin d'être isolé à cet égard : des concepts comme la matière, la vie ou encore, plus près de lui, l'environnement, le développement, sont eux aussi réinterprétés et n'ont pas la même signification en physique, en biologie ou encore en psychologie. Toulmin (en 1972) affirme d'ailleurs que c'est ce qui amène à considérer une situation de problèmes donnée comme vraiment problématique qui distingue une discipline scientifique d'une autre (cité par Nadeau, 1999, p. 167).

Si l'on prend l'exemple de l'environnement, on constate que celui-ci se trouve aussi décliné, par exemple dans des intitulés de revues scientifiques, selon un certain nombre de disciplines : sciences de l'environnement, sociologie de l'environnement, psychologie de l'environnement, économie de l'environnement, etc., mais qu'il est également associé à des thèmes traités par des disciplines, comme l'aménagement du territoire, la santé, etc. Il apparaît alors que l'un des destins du développement durable en matière de recherches est de connaître une évolution du même type : visions disciplinaires et corrélation avec différents thèmes d'études.

Qu'en est-il d'autres critères associés par Kuhn à une discipline? Il existe un certain nombre de revues scientifiques utilisant le terme de sustainability, ou de sustainable development. On y voit dominer dans la teneur des articles des contenus touchant au champ de l'environnement. Néanmoins, certaines revues et certains articles incluent aussi le champ plus large d'interprétation du développement durable ${ }^{7}$.

Des communautés de scientifiques peuvent être recensées, se référant à des revues, des congrès, des programmes de recherche relatifs au développement durable. Toutefois, ils ne s'y réfèrent pas exclusivement. Ainsi, si un article scientifique de science physique s'appuiera dans ses références sur d'autres ouvrages de cette discipline, ce

\footnotetext{
7 Si les sciences de la nature se retrouveront plutôt dans la première configuration, les sciences humaines peuvent embrasser les deux sens. Ainsi, dans le recueil Sustainability and the Social Sciences (Becker et Jahn, 1999), la plupart des contributions comprennent l'enjeu posé comme étant l'apport des sciences humaines à des questions écologiques. Mais certaines élaborent aussi sur la «social sustainability " (I. Sachs) ou sur une «transdisciplinarité autoréflexive » (Becker et al., 1999) sur les acteurs qui influencent les savoirs.
}

n'est pas le cas en développement durable où des travaux menés hors de ce champ sont couramment utilisés. En outre, ces communautés, bien que fédérées de façon large, sont scindées, en particulier à travers des disciplines ou des programmes de recherche s'adressant de façon variée à des problèmes de développement durable.

En matière de «plus haut diplôme obtenu », qui est un autre critère de constitution d'une discipline pour Kuhn, le développement durable n'est pas bien pourvu à l'heure actuelle. Il y a encore peu de diplômes ainsi estampillés dans les universités, bien qu'un mouvement existe pour les multiplier (voir pour la Grande-Bretagne Dawe et al., 2005). On recense plutôt un accroissement du nombre de cours traitant de divers sujets reliés au développement durable, insérés dans des formations existantes. Certaines de ces configurations conduisent à des spécialités disciplinaires (voir, ci-dessous, «L'enseignement du développement durable »).

\section{Paradigmes et révolutions}

Le développement durable ne peut donc être identifié à une discipline, bien qu'il en ait certains attributs. Peut-il être considéré comme un paradigme au sens de Kuhn? Si nous revenons aux définitions pratiques énoncées cidessus, qu'en serait-il de règles communes qui s'imposent à un ensemble de savants, ou de solutions concrètes que les étudiants rencontrent dès le début de leur formation?

Au niveau général, on peut difficilement retenir « la durabilité » comme concept candidat à constituer une telle « règle commune ", en raison de son flou et de ses contradictions internes ${ }^{8}$. On peut songer aussi à retenir des séries de principes associées au développement durable, comme l'équilibre des trois piliers, les principes de la déclaration de Rio ou d'autres encore. Mais, dans ce cas, on se heurte à l'écueil de la multiplicité des séries de principes existantes ${ }^{9}$. Même si l'on adopte des séries plus petites et plus centrales de critères, comme les trois critères cités plus haut rapportés spécifiquement à la recherche (temps long, espace élargi, interconnexions), il reste que la problématisation du développement durable sera, comme

\footnotetext{
8 Durabilité environnementale d'abord, évidemment, avec la figure d'une planète à préserver, mais qui à l'examen se ramifie en une multitude de problèmes divers, incluant la transformation de l'environnement. Durabilité aussi avec d'autres connotations multiformes: durabilité de compagnies commerciales, durabilité de sociétés riches face à l'émergence de concurrents, durabilité des systèmes de protection sociale, etc. Remarquons d'ailleurs que la durabilité n'est pas automatiquement une condition positive d'un point de vue humain : le désert ou, dans un autre sens, l'injustice peuvent êtres durables sans pour cela, évidemment, être des modèles de société.

${ }^{9}$ Le site http://www.iisd.org/sd/principle.asp de l'International Institute for Sustainable Development recense des dizaines de listes de principes, appliqués à différentes sphères (politique, économique, scientifique...) et différents niveaux.
} 
on l'a déjà noté, différente en fonction des disciplines. En outre, pour appliquer ces critères, les disciplines disposent de ressources très variables. Ainsi, par exemple, pour le temps long (en direction du futur), l'économie ou la climatologie disposent de modèles et de pratiques bien différents de ceux de la chimie ou de la biologie (et différents entre eux).

On est ainsi conduit à considérer ce qui se passe à l'intérieur de disciplines particulières. Dans certaines d'entre elles, on peut effectivement observer la constitution de règles ou d'exemples typiques - des paradigmes dans le sens vu ci-dessus - relatifs à des problèmes de soutenabilité. C'est particulièrement le cas en économie ${ }^{10}$, avec le courant Ecological Economics ou Environmental Economics, qui fédèrent $\mathrm{d}$ 'ailleurs des communautés de scientifiques en associations. On trouve d'autres exemples, ainsi en géographie (O'Riordan, 2004) ou en architecture, où de nouveaux objets avec des règles particulières fédèrent des questions de recherche : ville durable, bâtiments durables ${ }^{11}$.

À l'évidence, il existe à l'égard du développement durable une variété de positionnements parmi les disciplines et à l'intérieur de celles-ci (via des problèmes, des écoles et des questionnements variés). Plusieurs problèmes généraux sont posés, parmi lesquels, d'un côté, la définition de critères de développement durable (et leur mesure), de principes et de trajectoires pour y conduire (recherches sur le développement durable), et, d'un autre, l'innovation et l'application de techniques et de pratiques (y compris sociales) favorables à ce projet (recherches pour le développement durable). On peut ajouter, dans une troisième catégorie, l'analyse « méta » des transformations sociales en rapport avec le développement durable (qui incluent les concepts, discours, stratégies, etc.). Certaines disciplines sont plus présentes dans la recherche sur certains de ces problèmes, mais des variétés existent. Ainsi, par exemple, la biologie peut être appliquée pour mieux comprendre certains critères de durabilité, mais aussi pour développer des solutions techniques.

Assiste-t-on dans certains cas à des révolutions scientifiques ou des micro-révolutions au sens de Kuhn, autrement dit au fait que certains des rapports entre les objets étudiés changent, et que des faits se retrouveraient groupés dans des ensembles scientifiques différents? Il semble que l'on puisse répondre par l'affirmative selon deux axes de changements : changements internes de paradigmes; changements liés à la multidisciplinarité. On a déjà donné des exemples en ce qui concerne le premier axe, et le second sera examiné plus loin (voir

\footnotetext{
10 À cet égard, le petit livre de Harribey (1998) pourrait faire figure de manuel.

11 Plus largement pour les sciences sociales, voir Becker et al. $(1999$, p. 3) : «[...] sustainability is envisaged as a generator of problems, introducing a new and innovating type of questions for the social sciences, rather than providing a unifying paradigm. »
}

«Multidisciplinarité et programmes de recherche »). Ces changements affectent des groupes de scientifiques de tailles variables. Il n'est pas nécessaire, pour être qualifiés de révolutions en ce sens, qu'ils affectent l'ensemble de la discipline, même si les «convertis » appellent de leurs vœux leurs collègues à les rejoindre. Il n'est pas nécessaire non plus, d'ailleurs, que les disciplines aient connu des révolutions de paradigme pour faire des contributions utiles et pertinentes pour un développement durable ${ }^{12}$.

\section{Élargissement de l'analyse : les composantes politiques, sociales et institutionnelles}

L'analyse que nous avons tentée jusqu'ici n'est pas complète pour plusieurs raisons. D'abord, il existe une puissante composante politique qui ne peut être passée sous silence quand on traite du développement durable, même si on se place sur le terrain des recherches et des disciplines scientifiques. Elle peut d'autant moins être omise que les philosophes et les sociologues des sciences postkuhniens ont mis en évidence - pour les sciences en général - des interactions intimes entre discours et pratiques scientifiques, d'une part, et volet institutionnel, politique et social, de l'autre. Ceci va conditionner le concept même de discipline, en même temps que les frontières entre celles-ci auront moins d'intangibilité. Enfin, ces considérations auront des conséquences en matière de multidisciplinarité. Envisageons successivement ces différents points.

\section{Les conséquences de la vision politique sur les définitions}

Si le développement durable était une mythologie (et il l'est un peu en un sens), ses deux grands ancêtres, encore bien actifs, seraient l'environnement et le développement. Notons que le premier a une dominante dans les sciences naturelles et le second, dans les sciences humaines, ce qui offre au développement durable l'atout - ou la difficulté (Redclift, 1999) - de combiner les deux. Mais, par ailleurs, l'environnement et le développement ont aussi, joints à leur facette scientifique, des discours politiques insistants de changement, voire de révolution, et cette fois plus seulement au sens scientifique du terme.

\footnotetext{
12 Ajoutons qu'il n'est pas nécessaire non plus de se placer sous l'égide du développement durable pour appeler à de nouveaux paradigmes, à des approches multidisciplinaires, et à des révolutions épistémologiques combinant sciences de la nature et de l'homme. Edgar Morin en est un exemple évident, qui publiait déjà en 1973 Le Paradigme perdu : la nature humaine tout en se distanciant plus tard du développement durable qu'il critique pour son manque de rupture avec le développement techno-économique occidental.
} 
Lorsque l'on se penche sur la généalogie du développement durable, il apparaît que c'est dans cette dimension politique $^{13}$ au sens large, plus que dans les disciplines scientifiques, qu'il faut rechercher ce que l'on pourrait appeler par analogie sa matrice. Certes, les scientifiques ont contribué à fournir des constats alarmants sur des dégradations environnementales, mais c'est quand ceuxci sont entrés dans un registre politique, englobant, et mélangés en diverses mesures avec des constats et des questions éthiques sur le développement humain, que s'est constitué le registre du développement durable.

Quoi qu'il en soit, c'est principalement dans le champ sociopolitique que va se situer une dynamique tendant à imposer le développement durable comme une innovation positive, caractéristique que nous avons déjà notée comme présente dans la notion. Cela est visible dans des discours institutionnels de ces dernières années, dans lesquels les définitions précises sont moins importantes que les registres d'arguments, les visions du monde ou la légitimité des acteurs. De ce fait, en dépit de son défaut de cohérence, il existe des mécanismes puissants qui procurent une certaine unité au développement durable, et qui suscitent envers lui une adhésion spontanée. Sur le plan formel, ce sont des présentations de l'ordre du récit - des story lines (Hajer, 1995), selon le terme anglais, ce que l'on pourrait traduire par des «scénarisations »que le champ de l'environnement et du développement fournissent avec force ${ }^{14}$.

\section{Le développement durable comme méthode et éthique pour des chercheurs}

Irriguées de prescriptions et de contextes politiques, les recherches en développement durable s'avèrent particulièrement sensibles à ces dynamiques. Mais, en outre et plus généralement sur le plan théorique, des travaux récents de sociologie des sciences remettent en question la notion de cohérence disciplinaire elle-même (y compris donc hors développement durable). «Les disciplines seraient assimilables à des "formations discursives" qui garantissent la régularité de la coexistence de configurations d'éléments hétérogènes. Elle se forment par la stabilisation progressive d'une toile faite d'éléments hétérogènes, de déclarations issues de différents domaines,

\footnotetext{
13 Vivien (2005, p. 7) partage ce point de vue : «Le développement soutenable apparaît d'abord comme une notion politique. » Quant à Jollivet (2001), il parle d'une « problématique hybride » : "notion de recherche et catégorie pour l'action».

14 On peut relire à cet égard la belle réussite du premier chapitre du rapport Brundtland (CMED, 1987). Forsyth (2003) détaille longuement comment, dans l'écologie politique, des intrications entre mots d'ordre politiques et analyses scientifiques peuvent en arriver à poser problème quant à la validité des constructions scientifiques écologiques et à la pertinence de certaines prescriptions qui en découlent.
}

de différents types de discours », écrit par exemple Klein (2000, p. 401) en s'inspirant ici de Foucault, dans une vision qui pourrait être davantage d'application dans les sciences humaines. L'influence des institutions, de l'historicité et du contexte des communautés scientifiques est également fort soulignée - déjà chez Toulmin (1972), cité par Nadeau (1999, p. 168). Klein estime qu'en quelque sorte, «les disciplines sont finalisées lorsqu'elles adoptent des objectifs extérieurs : réponse à la demande sociale ou résolution de problèmes concrets » (Klein, op. cit.). Selon De Certaines (1976, cité par Klein, op. cit., p. 405), les disciplines seraient en elles-mêmes instables et seules « les contraintes externes les font paraitre comme des totalités stables ».

Pourrait-on, en phase avec ces analyses, considérer que le développement durable appellerait, comme "contrainte externe », de nouvelles pratiques scientifiques, voire des changements disciplinaires d'un ordre particulier? C'est ce que fait une chercheuse et philosophe des sciences comme Stengers (1998, p. 6), qui écrit que, «si le développement durable appelle une "nouvelle approche", ce n'est pas parce qu'il s'agit d'un "nouveau" problème [...]. Mais s'il doit y avoir une "nouvelle approche", c'est notamment parce que ces aspects nouveaux du problème ne disent pas en eux-mêmes comment ils doivent être pris en compte, comment ils doivent intervenir dans la caractérisation de ce que serait un développement durable». Ce qui conduit clairement, pour l'objet qui nous occupe ici, à des changements disciplinaires : "Il s'agirait que chacun précise ce que le développement durable impose de modifier dans les habitudes de recherche et les cultures disciplinaires » (ibid.).

À travers différentes variations, ce type de réflexion peut amener à une vocation particulière dans la configuration des recherches que suscite le développement durable : il ne serait plus tant un objet incluant tel ou tel contenu, mais une approche différente de l'inscription des scientifiques dans leur rôle, dans leur métier, dans les débats publics, face à la demande sociale. Pour Stengers (1998), des points-clés seraient de demander aux chercheurs d'étudier les objets de façon à montrer leurs interactions avec différents savoirs et avec les processus de décisions, de ne pas se désintéresser du devenir de leurs productions, en somme. En ce qui concerne la géographie, O'Riordan (2004, p. 234) écrit : « Geographers should neither be ashamed nor wary of entering into the political worlds of government, business and civil activism [...]. We now live in a world of coproduction of scientific and policy knowledge with actual behaviour. » Le rapport français sur la recherche pour un développement durable, déjà cité, indique sous l'intertitre « Vers un renforcement de la légitimité sociale des choix de la recherche scientifique et technologique »: «Pour répondre aux nouvelles exigences de visibilité publique de la recherche et aux nouvelles attentes concernant la maîtrise des retombées du développement scientifique, 
un soin renouvelé doit être porté à la définition des priorités : la légitimité et la pertinence scientifiques se voient confrontées à la légitimité politique » (Hautcœur, 2003, p. 12). On trouve dans cette perspective des appels à l'interaction avec les « utilisateurs » de recherche sous différentes formes ${ }^{15}$, pouvant déboucher, comme l'affirment Becker et al. (1999, p. 5), sur une " transdisciplinarité autoréflexive ", transdisciplinarité étant entendue comme s'étendant à des acteurs hors du monde scientifique.

\section{Multidisciplinarité et programmes de recherche}

Ayant pris la notion de discipline comme fil rouge de cette contribution, nous pouvons poursuivre cette réflexion en observant la dimension multidisciplinaire, souvent associée aux recherches rapportées au développement durable. On notera tout d'abord que ce caractère se trouve déjà être le fait des études environnementales, ainsi que de celles de développement, et il n'est pas surprenant de le retrouver ici.

Mais, plus largement, les évolutions scientifiques de ces dernières décennies ont rendu les approches multidisciplinaires plus courantes que par le passé dans beaucoup de domaines, via les réseaux de recherche par exemple (Mulkay et al., 1975, cité par Klein, 2000, p. 405). Le «mode 2 » de transmission de savoirs, centré sur les interrelations entre plusieurs sphères scientifiques, politiques, économiques - dont nous venons de noter l'importance dans le cadre du développement durable - gagne du terrain par rapport au "mode 1 », centré sur les disciplines académiques au sein de l'Université (selon Gibbons et al., 1994, ibid.). Les disciplines, avec leur découpage académique stable, deviennent dès lors moins cruciales pour la transmission des savoirs, notamment du fait du fonctionnement de ces programmes de recherche analysant des objets de façons multiples (Billaud, 2003), et aussi de dispositifs encourageant, dans un but d'application, les transmissions de savoirs entre sphères scientifiques, politiques, économiques, industrielles.

On peut comprendre dès lors que certains programmes de recherche récents, nationaux ou internationaux, cherchent à influencer volontairement la configuration même des recherches qu'ils financent, en exigeant une production et une mise en forme des savoirs selon certains critères définis à partir du développement durable $\mathrm{e}^{16}$. Par rapport aux disciplines, ces critères auront notamment

\footnotetext{
15 Billaud (2003), dans ce sens, parle de coproduction des savoirs.

16 Voir, par exemple, le programme Agriculture et développement durable (ADD), en France. Reste à savoir quelle sera la force de ces programmes de recherche publics quand de nombreuses autres influences s'exercent également sur la recherche : recherche finalisée industrielle liée à certains intérêts de court terme peu «soutenables », course aux brevets privés, ou encore déséquilibres des potentiels entre les nations riches
}

pour effet de revoir certains «cadrages » classiquement considérés comme valides, mais dont le développement durable signe une nécessaire prise en compte : «oubli » de l'environnement dans le socioéconomique, «oubli » des flux matériels dans l'économie, etc. ${ }^{17}$, ou, encore une fois, temps longs, espaces élargis, interconnexions. Cadrages, mais aussi éthiques disciplinaires, qui peuvent d'ailleurs être vues aussi comme des recadrages, mais dans un sens plus large (transdisciplinaire), incluant l'activité scientifique dans son contexte et ses finalités.

Godard (2001, p. 62) considère dans ce sens que «l'inscription de la recherche sous l'égide du développement durable conduirait à faire de la recherche autrement; concepts, méthodologies et pratiques seraient touchés; cela pourrait se faire à travers la médiation d'une éthique de la recherche ou, de façon plus directe, à travers le changement de visée de l'activité de recherche qui en modifierait toutes les conditions : il pourrait en résulter l'attribution d'une place plus importante à des dispositifs de recherche finalisée et transdisciplinaire conçus en fonction de la résolution de problèmes transversaux ». La Revue Durable présente aujourd'hui en français des articles et des dossiers de qualité qui illustrent ce type de perspective et de position.

\section{L'enseignement du développement durable}

Nous pouvons enfin tenter de tirer de ces analyses et de ces hypothèses quelques conséquences en ce qui concerne les relations entre enseignement et développement durable, en nous limitant au niveau universitaire. Ce domaine, lui aussi à l'intersection entre champs scientifique et politique (de l'éducation), a vu fleurir nombre de réflexions, initiatives et programmes, de la déclaration de Talloires de 1'Association of University Leaders for a Sustainable Future (USLF), datant de $1990^{18}$, à la Décennie pour l'éducation en vue du développement durable (2005-2014) des Nations unies.

Comme on l'a déjà noté en introduction, vu les structurations des programmes et les habitudes académiques, les évolutions éventuelles devront se situer le plus souvent dans le cadre d'enseignements disciplinaires. Dans ces structures, un certain nombre de thèmes et de problèmes, voire de nouvelles règles et principes quand c'est pertinent (par exemple en économie de l'environnement), peuvent être traités. Ces inclusions se trouvent aujourd'hui à des stades divers, notamment selon les disciplines, comme en

économiquement et celles dites «en développement », entre autres.

17 Nous concluions notre ouvrage (Zaccaï, 2002) sur cet aspect de révision des cadrages, qui nous semble transversal à une série de problématiques de développement durable.

18 Elle était signée, en mars 2006, par 328 institutions, dont 240 sur le continent américain. 
témoigne une étude récente en Grande-Bretagne (Dawe et al., 2005). Bien entendu, l'enseignement multidisciplinaire sera également promu : examiner des questions liées au développement durable à travers différentes approches et disciplines sera un passage obligé pour les enseigner (même si, dans la pratique, cela n'est sans doute pas si courant).

S'il n'est pas réellement une discipline, on pourrait soutenir que le développement durable peut alors devenir, dans certains cas, une spécialité pour des scientifiques diplômés en différentes disciplines. Les spécialités délimitent des « groupes de scientifiques qui travaillent sur des problèmes similaires » (Zuckerman, 1988, cité par Klein, 2000, p. 400). On constate que certains diplômes universitaires offrent maintenant effectivement des spécialités en développement durable.

Il va de soi que, dans l'enseignement aussi, les conceptions du développement durable et de ce qu'il implique sont multiples ${ }^{19}$. Ici aussi on trouvera une palette qui va de thèmes à fortes connotations environnementales, à un appel à une modification des méthodes d'enseignement, en passant par des configurations hybrides. Si la déclaration de Talloires est clairement centrée sur l'environnement, The Sustainability Curriculum (Blewitt et Cullingford, 2004), manuel destiné aux universités publié quatorze ans plus tard, inclut des sujets d'environnement, d'économie, de sociologie, de géographie, de citoyenneté ou encore d'écoféminisme. Remarquons toutefois qu'il ne comporte pas de chapitre sur l'emploi, la culture, la santé ou l'alimentation, par exemple, tandis que d'autres programmes comprendront notamment la santé et l'alimentation ${ }^{20}$.

Du côté des remises en cause plus fondamentales, on peut lire dans le rapport de Dawe et al. $(2005$, p. 5) : « [Education for sustainable development] represents a major shift in the way students are taught and learn within the higher education sector. It requires a broader and more flexible approach to the development and teaching of academic disciplines. » Sterling (2001), par exemple, établit une combinaison significative entre une réflexion sur des fonctions de base de l'enseignement et «the need to educate for sustainability, community and peace in a turbulent and rapidly changing world» (cité par Bassey, 2002, p. 903). On trouve aussi plusieurs contributions en ce sens dans Filho (2005). L'angle et les finalités de l'enseignement du ou pour le développement durable peuvent aussi être discutés. Ainsi, Jickling (1994) s'oppose à ce que l'enseignement soit dominé par des prescriptions et prône des cours visant à éclairer sur les

\footnotetext{
${ }^{19}$ Ce caractère pouvant même être revendiqué et accru, comme lorsque le site de la Décennie de l’Unesco propose : «Définissez le développement durable en 25 mots ou moins - veuillez rédiger et nous envoyer une phrase comprenant 25 mots maximum qui résume votre perception du développement durable. » (http://portal.unesco.org/education/fr/ev.php-URL_ID=27279).

20 Site http://www.eduquer-au-developpement-durable.com/, mais qui n'est pas destiné aux universités.
}

débats et les positions dans la problématique du développement durable. Quant à Stengers (1998), elle insiste sur l'enseignement de multiples cas de développements non durables comme de précieux éléments pour aider à la construction de projets qui puissent l'être davantage.

\section{Conclusions}

Le parcours que nous avons essayé de tracer a cherché à placer quelques repères face aux demandes relatives à l'inclusion de davantage de références au développement durable dans les milieux académiques en matière de recherche et d'enseignement. Le fil rouge des « disciplines scientifiques » a été choisi en raison de son caractère essentiel pour structurer les formations et les orientations scientifiques dans le monde académique. En matière de recherche, l'interrogation est venue se situer dans une évolution large, particulièrement visible en environnement, mais sans s'y limiter, dans laquelle multidisciplinarité et interactions entre scientifiques et acteurs politicosociaux jouent des rôles majeurs, même si les méthodes pour leur contribution à des résultats scientifiques posent des questions encore non résolues par rapport à des schémas plus traditionnels. Mais ces derniers ont posé également une série de problèmes, et leur réalité historique elle-même (stabilité des différences disciplinaires, autonomie de la production scientifique) est aujourd'hui relativisée. Par ailleurs, il est frappant de constater (sur l'exemple anglais) que dans l'enseignement également des changements sont suggérés dans des sens similaires pour modifier les transmissions de savoirs.

Le développement durable apparaît alors comme l'une des figures assumant et portant ces types d'évolution actuelle. L'analyse nous montre que, malgré sa position dominante à se proclamer comme une force majeure d'innovation en ce sens, il n'est sans doute que l'une des possibilités traduisant cette évolution.

En tout état de cause, c'est dans les textures mêmes des productions scientifiques et des enseignements que des changements peuvent se produire de façon diversifiée. Nous avons tenté de repérer des exemples selon certaines spécificités disciplinaires, avec des réorganisations parfois à l'œuvre, et d'autres qui seraient souhaitables. Ce caractère à la fois diversifié des concrétisations, en même temps que l'inclusion dans une référence transversale aux disciplines, apparaît comme une donnée très actuelle de l'état d'implémentation du développement durable dans le monde académique. Nous avons suggéré que les «spécialités » en développement durable accolées à certains diplômes pouvaient sans doute mieux correspondre aux évolutions que le fait de mettre en place une éventuelle discipline en cette matière.

Il n'est pas évident de trouver une juste distance par rapport aux invocations répétées à l'innovation portées 
par la force symbolique du développement durable. On ne peut rester sourd aux changements évidents exigés dans l'approche de certains problèmes majeurs, et d'ailleurs aussi mineurs. Mais on ne peut pour autant espérer que ce soit la multiplication des connexions et des sujets entrepris pour englober les multiples aspects d'une notion, dont on a vu les difficultés, qui par elle-même créerait l'approche souhaitée. L'orientation défendue ici est bien un examen fin en utilisant les outils intellectuels existants pour analyser l'irruption et l'influence du développement durable sur ces scènes académiques, en se réservant donc le temps de l'examen au cas par cas, avant de souscrire à l'affirmation générale d'innovation structurante qu'il peut porter.

\section{Remerciements}

Mes remerciements vont à Tom Bauler, Grégoire Wallenborn et Nathalie Zaccaï-Reyners pour leurs commentaires et leurs suggestions de références à consulter, ainsi qu'à Isabelle Stengers pour avoir souligné quelques points-clés. Olivier Godard a relancé mon intérêt pour cette réflexion grâce à de généreux commentaires sur une première version du texte. Le rapport de lecture de la revue NSS, et spécialement de Bernard Hubert, m'a encore aidé à recadrer la version finale. Bien entendu, les idées ici exposées n'engagent que moi-même.

\section{Références}

Adams, W.M., 2001. Green Development: Environment and Sustainability in the Third World, Londres, Routledge.

Aubertin, C., Vivien, F-D. (Eds), 2006. Le Développement durable : enjeux politiques, économiques et sociaux, Paris, La Documentation française.

Audi, R. (Ed.), 1995. The Cambridge Dictionary of Philosophy, Cambridge, Cambridge University Press.

Bassey, M., 2002. Sustainable Education: Re-Visioning Learning and Change, Book Reviews, British Educational Research Journal, 28, 6, 903-904.

Becker, E., Jahn, T., 1999. Sustainability and the Social Sciences: A Cross-disciplinary Approach to Integrating Environmental Considerations into Theoretical Reorientation, London, Zed Books.

Becker, E., Jahn, T., Stiess, I., 1999. Exploring uncommon ground: Sustainability and the social sciences, in Becker, E., Jahn, T., Sustainability and the Social Sciences: A Cross-disciplinary Approach to Integrating Environmental Considerations into Theoretical Reorientation, London, Zed Books, 1-22.

Billaud, J.-P., 2003. De l'objet de l'interdisciplinarité à l'interdisciplinarité autour des objets, Natures Sciences Sociétés, 11, 1, 29-36.

Blewitt, J., Cullingford, C. (Eds), 2004. The Sustainability Curriculum: The Challenge for Higher Education, London, Earthscan.

Commission des Communautés européennes, 2002. Communication de la Commission sur l'analyse d'impact, Bruxelles, COM(2002) 276 final.
Commission mondiale sur l'environnement et le développement (CMED), 1987. Notre avenir à tous (rapport Brundtland), New York, Nations unies; Québec, Éditions du Fleuve / Publications du Québec, 1988.

Dawe, G., Jucker, R., Martin, S., 2005. Sustainable Development and Higher Education: Current Practice and Future Development. A report to the Higher Education Academy, York (UK) (http://www.heacademy.ac.uk/assets/York/documents/ ourwork/tla/sustainability/sustdevinHEfinalreport.pdf).

Certaines, J. de, 1976. La biophysique en France : critique de la notion de discipline scientifique, in Lemaine, G., et al., Perspectives on the Emergence of Scientific Disciplines, Paris, Mouton / Chicago, Aldine.

Filho, W. (Ed.), 2005. Handbook of Sustainability Research, Bern, Frankfurt am Main, Peter Lang.

Forsyth, T., 2003. Critical Political Ecology, London, Routledge.

Gibbons, M., et al., 1994. The New Production of Knowledge, London, Sage.

Godard, O., 2001. Le développement durable et la recherche scientifique, ou la difficile conciliation des logiques de l'action et de la connaissance, in Jollivet, M. (Ed.), Le Développement durable, de l'utopie au concept : de nouveaux chantiers pour la recherche, Paris, Elsevier, 61-81.

Hajer, M., 1995. The Politics of Environmental Discourse: Ecological Modernization and the Policy Process, Oxford, New York, Clarendon Press.

Harribey, J.-M., 1998. Le Développement soutenable, Paris, Economica.

Hautcœur, P.-C. (Rapporteur), 2003. La Recherche au service du développement durable. Rapport intermédiaire, ministère délégué à la Recherche et aux Nouvelles technologies / secrétariat d'État au Développement durable, Paris (http:// 160.92.130.199/rapport/devdurable/ddinter.pdf). Publication finale en 2005, Paris, La Documentation française.

Hopwood, B., Mellor, M., O’Brien, G., 2005. Sustainable Development: Mapping Different Approaches, Sustainable Development, 13, 38-52.

Jickling, B., 1994. Studying Sustainable Development: Problems and Possibilities, Canadian Journal of Education, 19, 3, 231-240.

Jollivet, M., 2001. Le développement durable, notion de recherche et catégorie pour l'action. Canevas pour une problématique hybride, in Jollivet, M. (Ed.), Le Développement durable, de l'utopie au concept: de nouveaux chantiers pour la recherche, Paris, Elsevier, 97-116.

Klein, G., 2000. L'aventure des disciplines. Trois thèses dans les études de la science contemporaine, Cahiers internationaux de sociologie, CIX, 393-414.

Kuhn, T., 1983. La Structure des révolutions scientifiques, Paris, Flammarion.

Mamou, Y., 2006. Les grands groupes font du développement durable un outil de productivité, Le Monde, 5 septembre, 13.

Morin, E., 1973. Le Paradigme perdu : la nature humaine, Paris, Le Seuil.

Mulkay, M., Gilbert, G., Woolgar, W., 1975. Problem Areas and Research Networks in Science, Sociology, 9, 187-203.

Nadeau, R., 1999. Vocabulaire technique et analytique de l'épistémologie, Paris, PUF.

Newton, J., Freyfogle, E., 2005. Sustainability: a Dissent, Conservation Biology, 19, 1, 23-32.

O'Riordan, T., 2004. Environmental science, sustainability and politics, Transactions of the Institute of British Geographers, 29, 234-247. 
Redclift, M., 1999. Dance with wolves? Sustainability and the social sciences, in Becker, E., Jahn, T., Sustainability and the Social Sciences: A Cross-disciplinary Approach to Integrating Environmental Considerations into Theoretical Reorientation, London, Zed Books, 267-273.

Reid, D., 1995. Sustainable Development: An Introductory Guide, London, Earthscan.

Smouts, M.-C. (Ed.), 2005. Le Développement durable : les termes du débat, Paris, Armand Colin.

Stengers, I., 1998. Prendre au sérieux le développement durable?, Cahiers du CEDD, 3 (www.ulb.ac.be/igeat/cedd). Publié également sous le titre : Le développement durable : une nouvelle approche?, Alliage, 40 (1999), 31-39.
Sterling, S.R., 2001. Sustainable Education: Re-Visioning Learning and Change, Totnes, Green Books for the Schumacher Society.

Toulmin, S., 1972. Human Understanding, 1. The Collective Use and Evolution of Concepts, Princeton, Princeton University Press.

Vivien, F.-D., 2005. Le Développement soutenable, Paris, La Découverte.

Zaccaï, E., 2002. Le Développement durable : dynamique et constitution d'un projet, Bern, Bruxelles, Peter Lang.

Zuckerman, H., 1988. The sociology of science, in Smelser, N.J., Handbook of Sociology, Newbury Park, Sage Publications.

Reçu le 6 novembre 2005. Accepté le 6 septembre 2007. 